\title{
Real-world adherence and persistence with anaplastic lymphoma kinase inhibitors in non-small cell lung cancer
}

\author{
Apar Kishor Ganti, MD; Chia-Wei Lin, PhD; Erru Yang, MS; William B Wong, PharmD, MS; and Sarika Ogale, PhD
}

\section{What is already known about this subject}

- Several anaplastic lymphoma kinase (ALK) inhibitors have been approved for the treatment of metastatic ALK-positive non-small cell lung cancer (NSCLC), including alectinib, crizotinib, brigatinib, ceritinib, and lorlatinib.

- While efficacy and safety are well documented, real-world data on the use of ALK inhibitors are currently limited.

\section{What this study adds}

- In this pooled analysis of 3 US insurance claims databases, alectinib was associated with longer real-world persistence than other ALK inhibitors, despite similar adherence.

- This is the first study to assess adherence and persistence with ALK inhibitors for the treatment of patients with ALK-positive NSCLC in real-world clinical practice.

\section{Author affiliations}

Apar Kishor Ganti, MD, VA Nebraska-Western lowa Health Care System, Omaha, NE, and University of Nebraska Medical Center, Omaha. Chia-Wei Lin, PhD; Erru Yang, MS; William B Wong, PharmD, MS; and Sarika Ogale, PhD, Genentech Inc., South San Francisco, CA.

\section{AUTHOR CORRESPONDENCE: \\ Chia-Wei Lin, 650.334.5594; \\ lin.chiawei@gene.com}

J Manag Care Spec Pharm 2022;28(3):305-14

Copyright $@ 2022$, Academy of Managed Care Pharmacy. All rights reserved.

\section{ABSTRACT}

BACKGROUND: Lung cancer is the leading cause of cancer-related deaths in the United States. Several anaplastic lymphoma kinase (ALK) rearrangement inhibitors have been approved for the treatment of metastatic ALK-positive non-small cell lung cancer (NSCLC). Effective disease management requires an understanding of how these treatments are used in clinical practice, since low treatment adherence and/or early discontinuation have been associated with poor patient outcomes. Owing to the recency of approvals, real-world data on the use of ALK inhibitors in patients with ALKpositive NSCLC are currently limited; this represents a notable gap in our understanding of ALK treatment use.
OBJECTIVE: To assess real-world adherence and persistence with ALK inhibitors in patients with ALK-positive NSCLC.

METHODS: This retrospective observational study used US commercial claims for patients aged at least 18 years with lung cancer receiving ALK inhibitors (alectinib, brigatinib, ceritinib, crizotinib) between July 1, 2015, and December 31, 2018. Patients' first and any subsequent ALK inhibitor uses were categorized into ALK inhibitor-naive and ALK inhibitor-pretreated cohorts, respectively. Adherence was measured by medication possession ratio and persistence by time from treatment initiation to discontinuation (earliest of a treatment switch or greater than a 60-day gap). Descriptive statistics were used to summarize patient characteristics. Cohort comparisons were made using chi-square tests and t-tests. Persistence and time to next
ALK inhibitor were analyzed using KaplanMeier methods and the log-rank test. Poisson and Cox regression models of adherence and persistence, respectively, were applied to compare ALK inhibitors.

RESULTS: We identified 1,482 patients treated with alectinib $(n=445)$ or crizotinib $(n=1,037)$ in the ALK inhibitor-naive cohort; 604,142 , and 134 patients received alectinib, brigatinib, or ceritinib in the ALK inhibitorpretreated cohort. Adherence during the treatment period (95\%-97\%) and the proportion of patients with a medication possession ratio of at least 0.8 (92\%-95\%) were similar for all ALK inhibitors. In the ALK inhibitor-naive cohort, median time to treatment discontinuation with alectinib and crizotinib was 27.1 and 8.8 months, respectively; patients receiving alectinib were $46 \%$ less likely to discontinue than patients receiving crizotinib 
(adjusted hazard ratio [aHR] [95\% Cl]: 0.54 [0.44-0.65]; $P<0.0001)$. In the ALK inhibitor-pretreated cohort, the discontinuation risk for alectinib was 64\% lower than for ceritinib (aHR [95\% Cl]: 0.36 [0.27-0.49]; $\mathrm{P}<0.0001$ ) and $34 \%$ lower than for brigatinib (aHR [95\% Cl]: $0.66[0.42-$ 1.02]; $P=0.062$ ).

CONCLUSIONS: To our knowledge, this study is the first to address a current research gap by assessing real-world adherence and persistence with ALK inhibitors among patients with ALK-positive NSCLC in real-world clinical practice. Alectinib was associated with longer real-world persistence than other ALK inhibitors, despite similar adherence. Further research with more patients and longer follow-up is needed to link persistence to real-world clinical outcomes.

Lung cancer is the second most common cancer type and the leading cause of cancer-related deaths in the United States. ${ }^{1,2}$ Approximately $85 \%$ of all lung cancers are classified as non-small cell lung cancer (NSCLC), ${ }^{3}$ of which $~ 5 \%$ have an anaplastic lymphoma kinase (ALK) rearrangement. ${ }^{4,5}$ Although the prognosis for patients with late-stage NSCLC remains poor, with a median survival of less than 1 year and with only $5 \%$ of patients surviving more than 5 years, ${ }^{6,7}$ prospects for patients with ALK-positive NSCLC have become more promising due to the availability of ALK inhibitors.

Crizotinib was the first ALK inhibitor to be approved by the US Food and Drug Administration for the treatment of patients with ALK-positive NSCLC, ${ }^{8}$ and it rapidly became the standard first-line treatment for this patient population. ${ }^{9}$ However, almost all patients develop resistance to crizotinib within 1-2 years of initiation, with the brain being a common site of metastasis. ${ }^{10,11}$ Since the launch of crizotinib, several next-generation ALK inhibitors (alectinib, brigatinib, ceritinib, and lorlatinib) have become available for the treatment of ALK-positive NSCLC.. ${ }^{12,13}$ Alectinib received approval for first-line use in 2017 and became the preferred first-line treatment option for patients with ALK-positive NSCLC following 3 phase 3 studies demonstrating superior efficacy to crizotinib and a favorable safety profile. ${ }^{14-16}$ In an updated analysis of the ALEX trial, the 5-year survival rate for alectinib was $62.5 \%$ vs $45.5 \%$ for crizotinib. ${ }^{14}$

Adherence measures patient compliance to prescribed medicines, whereas persistence is a measure of the duration of therapy. Because ALK inhibitors are indicated for use until there is evidence of disease progression or toxicity, persistence could also be considered an indicator of the efficacy and safety of these treatments. ${ }^{17}$ Low adherence to oral cancer therapies and/or early discontinuation are often associated with poor patient outcomes and high rates of hospitalization. ${ }^{18}$ However, limited real-world data on the use of these oral ALK inhibitors are currently available, and medication adherence and persistence have not been investigated. Therefore, this study aimed to assess real-world adherence to and estimate real-world persistence with ALK inhibitors. An improved understanding of adherence to and persistence with these treatments will help to guide disease management in patients with ALK-positive NSCLC.

\section{Methods}

\section{STUDY DESIGN AND DATA SOURCES}

This was a retrospective, observational study of adult patients (aged at least 18 years) with lung cancer treated with ALK inhibitors (alectinib, brigatinib, ceritinib, or crizotinib). To maximize the sample size, deidentified data were pooled from 3 US commercial claims databases: the IBM MarketScan Commercial Claims and Medicare Supplemental databases (IBM MarketScan), the IQVIA PharMetrics Plus claims database (PharMetrics Plus), and the Symphony Health Integrated Dataverse (IDV). IBM MarketScan and PharMetrics Plus are large insurance claims databases that cover approximately 150 million and 170 million individuals, respectively, and are considered representative of the commercially insured population. IDV is a nationally representative, provider-based, cross-sectional database of medical/pharmacy claims submitted to all payer types in the United States (no patient enrollment data are available); annually, IDV covers approximately 260 million lives. All datasets meet Health Insurance Portability and Accountability Act requirements; hence, this study did not require institutional review board approval.

\section{PATIENT SELECTION}

A summary of patient selection is shown in Figure 1. Eligible patients were required to have at least 1 pharmacy claim for an ALK inhibitor between July 1, 2015, and December 31, 2018. Patients in PharMetrics Plus and IBM MarketScan were also required to have a lung cancer diagnosis (International Classification of Diseases, Ninth Revision, Clinical Modification code 162 or Tenth Revision code C34) between January 1, 2015, and December 31, 2018, and between January 1, 2015, and September 30, 2018, respectively. For patients in the IDV, a lung cancer diagnosis code was not required. The index date for each ALK inhibitor was defined as the date of the first prescription in the treatment sequence (Supplementary Figure 1, available in online article). The first ALK inhibitor prescription was identified based on the absence of an ALK inhibitor claim during the 


\section{FIGURE 1 Patient Selection}

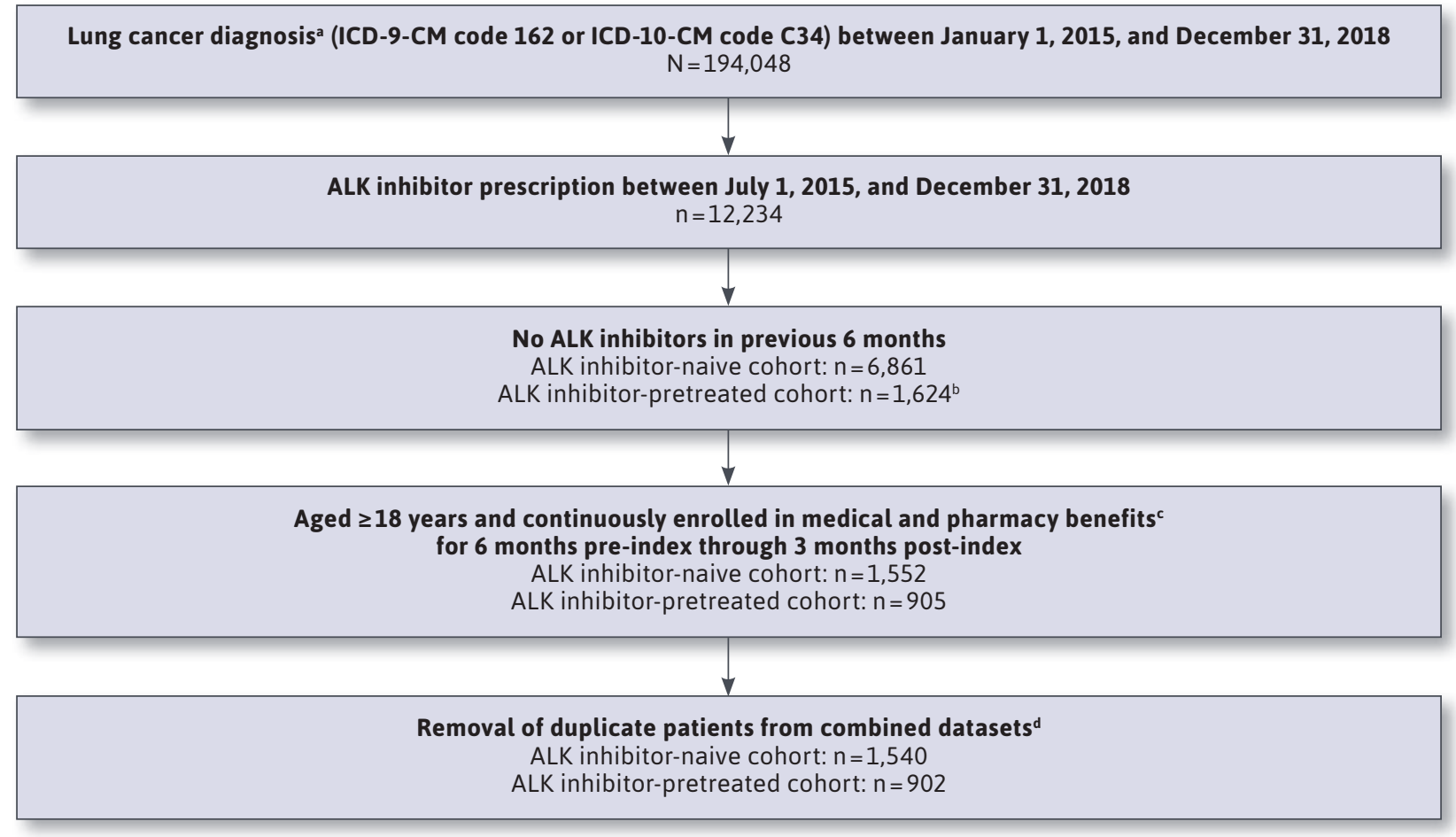

aDiagnosis not required in IDV; IBM MarketScan data only available up to September 30, 2018.

${ }^{b}$ Patients who had third and fourth ALK inhibitors had multiple observations.

'Enrollment data not available in IDV; instead, at least 1 claims activity during the period was required.

${ }^{d}$ De-duplication based on age, sex, index date, and index drug. For duplicate claims that were identified in IDV, data were taken from PharMetrics Plus or IBM MarketScan; no duplicates were found between PharMetrics Plus and IBM MarketScan.

$A L K=$ anaplastic lymphoma kinase; ICD-9-CM/ICD-10-CM = International Classification of Diseases, Ninth/Tenth Revision, Clinical Modification; IDV=Integrated Dataverse.

6 months before the index date. Patients in PharMetrics Plus and IBM MarketScan were required to have continuous enrollment in medical and pharmacy plans for at least 6 months pre-index and at least 3 months post-index. Since IDV does not have enrollment data, patients were required to have at least 1 medical claim and 1 pharmacy claim activity every 3 months in the 6 -month pre-index period and at least 1 non-index pharmacy claim at 3 months post-index to capture continuous activities in IDV.

For each patient, the date of the last pharmacy claim prior to the first gap between pharmacy fills was considered a surrogate for the end of follow-up. To address the potential overlap of different data sources, 14 duplicate patient records (identified by age, sex, index date, and first ALK inhibitor in the study period) were found for PharMetrics Plus or IBM MarketScan vs IDV. For these duplicates, patient data were taken from PharMetrics Plus or IBM MarketScan; no duplicates were found between PharMetrics Plus and IBM MarketScan.

Two cohorts were created based on ALK inhibitor use: the ALK inhibitor-naive cohort included data on the first observed use of an ALK inhibitor for each patient, and the ALK inhibitor-pretreated cohort included data on the second and all subsequent uses of ALK inhibitors for each patient (Table 1). The 2 cohorts were not mutually exclusive. Patients would contribute to more than 1 observation in the ALK inhibitor-pretreated cohort if they received a third or subsequent ALK inhibitor.

\section{OUTCOME MEASURES}

Adherence to each ALK inhibitor was assessed in patients with at least 2 prescriptions for that ALK inhibitor based on 


\section{TABLE 1 Baseline Characteristics of Eligible Patients Treated With ALK Inhibitors}

\begin{tabular}{|c|c|c|c|c|c|c|c|}
\hline & \multicolumn{3}{|c|}{ ALK inhibitor-naive cohort ${ }^{a}$} & \multicolumn{4}{|c|}{ ALK inhibitor-pretreated cohort $^{\text {b }}$} \\
\hline & $\begin{array}{c}\text { Alectinib } \\
n=445\end{array}$ & $\begin{array}{c}\text { Crizotinib } \\
n=1,037\end{array}$ & P value & $\begin{array}{c}\text { Alectinib } \\
n=604\end{array}$ & $\begin{array}{c}\begin{array}{c}\text { Brigatinib } \\
\mathrm{n}=\mathbf{1 4 2}\end{array} \\
\end{array}$ & $\begin{array}{c}\text { Ceritinib } \\
\mathrm{n}=134\end{array}$ & Pvalue $^{c}$ \\
\hline Age, years, mean (SD) & $58.9(11.7)$ & $60.7(12.7)$ & 0.002 & $56.6(12.2)$ & $55.0(12.5)$ & $56.8(12.8)$ & 0.484 \\
\hline \multicolumn{8}{|l|}{ Age category, years, n (\%) } \\
\hline $18-44$ & $53(11.9)$ & $121(11.7)$ & \multirow{5}{*}{0.001} & $103(17.1)$ & $24(16.9)$ & $24(17.9)$ & \multirow{5}{*}{0.515} \\
\hline $45-54$ & $101(22.7)$ & $163(15.7)$ & & $135(22.4)$ & $39(27.5)$ & $30(22.4)$ & \\
\hline $55-64$ & $158(35.5)$ & $356(34.3)$ & & $225(37.3)$ & $49(34.5)$ & $42(31.3)$ & \\
\hline $65-74$ & $88(19.8)$ & $223(21.5)$ & & $98(16.2)$ & $23(16.2)$ & $27(20.1)$ & \\
\hline$\geq 75$ & $45(10.1)$ & $174(16.8)$ & & $43 \quad(7.1)$ & $7 \quad(4.9)$ & $11 \quad(8.2)$ & \\
\hline \multicolumn{8}{|l|}{ Sex, n (\%) } \\
\hline Female & $255(57.3)$ & $585(56.4)$ & 0.751 & $316(52.3)$ & $74(52.1)$ & $65(48.5)$ & 0.723 \\
\hline $\mathrm{CCl}$ score, mean (SD) & $0.68 \quad(1.0)$ & $0.93 \quad(1.3)$ & 0.001 & $0.66 \quad(1.1)$ & $0.82 \quad(1.1)$ & $0.76 \quad(1.2)$ & 0.069 \\
\hline \multicolumn{8}{|l|}{$\mathrm{CCl}$ score } \\
\hline 0 & $262(58.9)$ & $521(50.2)$ & \multirow{3}{*}{0.006} & $395(65.4)$ & 78 (54.9) & 78 (58.2) & \multirow{3}{*}{0.068} \\
\hline 1 & $107(24.0)$ & $278(26.8)$ & & $87(14.4)$ & $29(20.4)$ & $29(21.6)$ & \\
\hline$\geq 2$ & $76(17.1)$ & $238(23.0)$ & & $122(20.2)$ & $35(24.6)$ & $27(20.1)$ & \\
\hline \multicolumn{8}{|l|}{ US region, $n(\%)$} \\
\hline Midwest & $100(22.5)$ & $255(24.6)$ & \multirow{5}{*}{0.804} & $150(24.8)$ & $32(22.5)$ & $40 \quad(29.9)$ & \multirow{5}{*}{0.100} \\
\hline Northeast & $105(23.6)$ & $232(22.4)$ & & $139(23.0)$ & $27(19.0)$ & $21(15.7)$ & \\
\hline South & $162(36.4)$ & 388 (37.4) & & $228 \quad(37.7)$ & $57(40.1)$ & $42(31.3)$ & \\
\hline West & 76 (17.1) & 157 (15.1) & & $85(14.1)$ & $26(18.3)$ & $31(23.1)$ & \\
\hline Unknown & $2 \quad(0.5)$ & $5 \quad(0.5)$ & & $2 \quad(0.3)$ & - & - & \\
\hline \multicolumn{8}{|c|}{ Health care insurance plan type, n (\%) } \\
\hline Individually purchased & $92(20.7)$ & 178 (17.2) & \multirow{5}{*}{0.017} & 120 (19.9) & $25(17.6)$ & $22(16.4)$ & \multirow{5}{*}{0.216} \\
\hline Employer-sponsored & $143(32.1)$ & 288 & & $210(34.8)$ & $41(28.9)$ & $48(35.8)$ & \\
\hline Medicaid & $18 \quad(4.0)$ & $66 \quad(6.4)$ & & $40 \quad(6.6)$ & $19(13.4)$ & $12 \quad(9.0)$ & \\
\hline Medicare & $101(22.7)$ & $303(29.2)$ & & $102(16.9)$ & $25(17.6)$ & $28(20.9)$ & \\
\hline Other/unknown & $91(20.5)$ & $202(19.5)$ & & $132(21.9)$ & $32(22.5)$ & $24(17.9)$ & \\
\hline \multicolumn{8}{|c|}{$\begin{array}{l}{ }^{a} A L K \text { inhibitor use was mutually exclusive in the ALK inhibitor-naive cohort. } \\
\text { bALK inhibitor use was not mutually exclusive in the ALK inhibitor-pretreated cohort. } \\
\text { cBold indicates a statistically significant } P \text { value. }\end{array}$} \\
\hline
\end{tabular}

medication possession ratio (MPR). MPR was defined as the number of days supply of ALK inhibitor medication divided by the total number of days between first prescription and last day of supply of the final prescription..$^{19}$ Persistence was estimated by the time from the index date to treatment discontinuation, which was defined as switching to another ALK inhibitor or a gap of at least 60 days without treatment with the same ALK inhibitor (whichever came first). Patients who disenrolled from their health care insurance plan or reached the end of the study period before treatment discontinuation were censored in the statistical analyses. A sensitivity analysis using a 30-day gap in the definition of persistence was conducted in both cohorts to assess the robustness of the analyses. An additional sensitivity analysis of time to next ALK inhibitor (defined as the time from index date of the first ALK inhibitor to index date of the next ALK inhibitor) was conducted for the ALK inhibitor-naive cohort. 


\section{TABLE 2 Treatment Adherence for Patients With at Least 2 ALK Inhibitor Prescriptions}

\begin{tabular}{|c|c|c|c|c|c|c|}
\hline & \multicolumn{2}{|c|}{ ALK inhibitor-naive cohort ${ }^{a}$} & \multicolumn{4}{|c|}{ ALK inhibitor-pretreated cohort $^{b}$} \\
\hline & Alectinib $(n=395)$ & Crizotinib $(n=885)$ & Alectinib $(n=566)$ & Brigatin & ib $(n=132)$ & Ceritinib $(n=120)$ \\
\hline \multicolumn{7}{|l|}{ Adherence $^{c}$} \\
\hline MPR, mean (SD) & $0.974(0.084)$ & $0.973(0.098)$ & $0.958(0.115)$ & \multicolumn{2}{|c|}{$0.965(0.122)$} & $0.974(0.089)$ \\
\hline Patients with MPR $\geq 0.8, \%$ & 95.2 & 94.9 & 91.7 & \multicolumn{2}{|c|}{93.2} & 92.5 \\
\hline At 3 months $^{d}$ & 80.2 & 70.9 & 83.1 & \multicolumn{2}{|c|}{78.2} & 76.9 \\
\hline At 6 months $^{\mathrm{e}}$ & $79.1(n=330)$ & $67.0(n=658)$ & $80.8(n=480)$ & \multicolumn{2}{|c|}{$70.5(n=78)$} & $60.8(n=74)$ \\
\hline \multicolumn{7}{|l|}{ Number of enrollment days ${ }^{f}$} \\
\hline Mean (SD) & $(224.0)$ & $(290.6)$ & $(267.6)$ & 245.3 & $(152.2)$ & $(307.5)$ \\
\hline Median (IQR) & $340.0(198-488)$ & $293.0(138-578)$ & $404.5(216-597)$ & 204.5 & $(142-315)$ & $242.0(113-599)$ \\
\hline \multicolumn{7}{|l|}{ Index treatment duration, days } \\
\hline Mean (SD) & $(220.1)$ & $(222.6)$ & $(265.2)$ & 195.6 & $(145.2)$ & $(186.9)$ \\
\hline Median (IQR) & $262.0(135-439)$ & $152.0 \quad(81-293)$ & $285.5(153-518)$ & 160.0 & $(92-263)$ & $121.0 \quad(70-257)$ \\
\hline Adjusted adherence $(M P R \geq 0.8)^{\mathrm{g}}$ & \multicolumn{2}{|c|}{ Alectinib vs crizotinib (ref) } & \multicolumn{2}{|c|}{ Alectinib vs brigatinib (ref) } & \multicolumn{2}{|c|}{ Alectinib vs ceritinib (ref) } \\
\hline $\operatorname{IRR}(95 \% \mathrm{Cl})$ & 1.00 & $(0.89-1.14)$ & $0.98 \quad(0.81-1$ & .20) & 0.9 & $(0.81-1.22)$ \\
\hline 3-month IRR $(95 \% \mathrm{Cl})$ & 1.12 & $(0.98-1.27)$ & $1.06 \quad(0.86-1$ & .31) & 1.0 & $(0.87-1.34)$ \\
\hline 6-month IRRe $(95 \% \mathrm{CI})$ & $\begin{array}{l}1.19 \\
\mathrm{n}=330\end{array}$ & $\begin{array}{l}(1.02-1.39) \\
1 \mathrm{~S} \mathrm{n}=658\end{array}$ & $\begin{array}{l}1.23 \quad(0.85-1 \\
\mathrm{n}=480 \text { vs } \mathrm{n}=\end{array}$ & & & $\begin{array}{l}(0.97-1.81) \\
480 \text { vs } n=74\end{array}$ \\
\hline \multicolumn{7}{|c|}{ aLK inhibitor use was mutually exclusive in the ALK inhibitor-naive cohort. } \\
\hline \multirow{2}{*}{\multicolumn{7}{|c|}{$\begin{array}{l}\text { CMPR is defined as the number of days of ALK inhibitor medication supplied divided by the total number of days between the first prescription and the last day of } \\
\text { supply of the final prescription (among patients with at least } 2 \text { prescriptions). } \\
{ }^{d} \text { Based on the total sample. }\end{array}$}} \\
\hline & & & & & & \\
\hline \multicolumn{7}{|c|}{$\begin{array}{l}\text { eCalculated for patients with } 6 \text { months of continuous enrollment post-index. For MarketScan and PharMetrics Plus, enrollment data were used; for IDV, medical and } \\
\text { pharmacy claims were used to determine continuous enrollment. }\end{array}$} \\
\hline \multirow{2}{*}{\multicolumn{7}{|c|}{$\begin{array}{l}\text { ISince IDV does not have enrollment data, patients were required to have at least } 1 \text { medical claim and } 1 \text { pharmacy claim activity every } 3 \text { months in the } 6 \text { months } \\
\text { pre-index and at least } 1 \text { non-index pharmacy claim within } 3 \text { months post-index to capture continuous activities in IDV. } \\
{ }^{9} \text { Adjusted for age, sex, CCI, region, health care insurance plan type, and indictor of the third or later ALK inhibitor (ALK inhibitor-pretreated model only) using }\end{array}$}} \\
\hline & & & & & & \\
\hline \multicolumn{7}{|c|}{$\begin{array}{l}A L K=\text { anaplastic lymphoma kinase; } C C I=\text { Charlson Comorbidity Index; IDV =Integrated Dataverse; IQR = interquartile range; IRR=incidence rate ratio; } \\
M P R=\text { medication possession ratio; ref = reference group. }\end{array}$} \\
\hline
\end{tabular}

\section{STATISTICAL ANALYSIS}

Descriptive statistics, including means, medians, and cohort proportions, were used to summarize patient characteristics; comparisons between cohorts were made using chi-square tests for categorical variables and t-tests for continuous variables. Mean MPR and the proportion of patients with an MPR of at least 0.8 were estimated for the entire treatment period and at 3 and 6 months post-index, if data were available. Persistence and time to next ALK inhibitor were analyzed using Kaplan-Meier methods and compared using the log-rank test. Poisson regression with log link for binary outcomes with an MPR of at least 0.8 and Cox regression models of adherence and persistence, respectively, were applied to compare alectinib with other ALK inhibitors after adjustment for baseline characteristics (sex, age, Charlson Comorbidity Index [CCI], US region, and insurance plan type) and sequence of ALK inhibitors (a binary indicator for the third or later ALK inhibitor was included in the model for the pretreated cohort). Note that no modification of Poisson regression with binary outcome was applied, and the overestimation of standard errors represented a more conservative interpretation of the findings. ${ }^{20}$

Incidence rate ratios (IRRs) and adjusted hazard ratios (aHRs) were reported with 95\% CIs. Patient characteristics and outcomes were analyzed separately for PharMetrics Plus and IDV and found to be consistent with the pooled analysis. Therefore, the pooled dataset was used as it increased sample sizes for each ALK inhibitor and enabled a meaningful comparison. SAS 9.4 (SAS Institute) was used to 


\section{FIGURE 2 Time to Treatment Discontinuation With Alectinib or Crizotinib in the ALK Inhibitor-Naive Cohort}

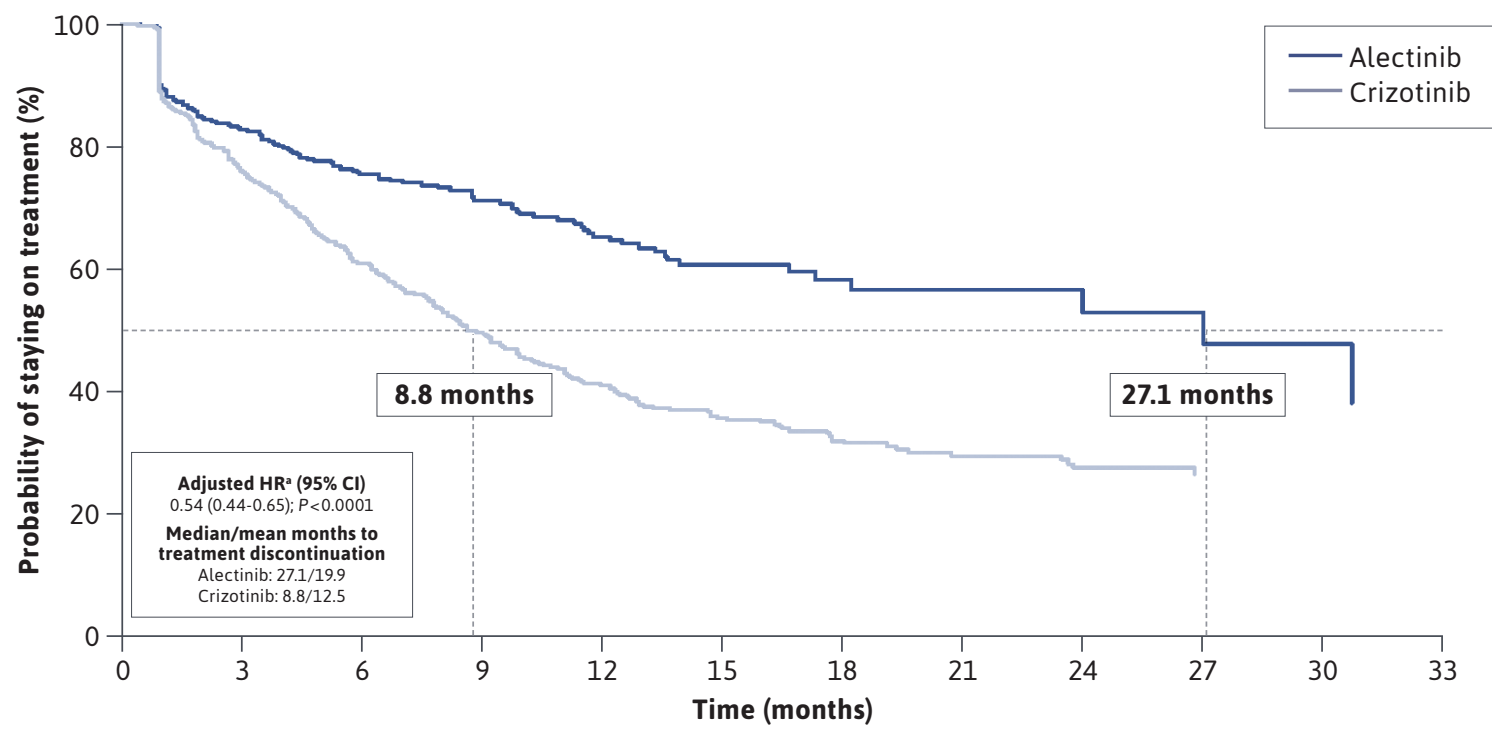

Patients on treatment $(\mathbf{n})$

\begin{tabular}{l|r|r|r|r|r|r|r|r|r|r|r|r}
\hline Alectinib & 445 & 338 & 235 & 166 & 120 & 87 & 43 & 38 & 38 & 14 & 9 & 4 \\
\hline Crizotinib & 1,037 & 629 & 385 & 243 & 166 & 116 & 79 & 53 & 41 & 30 & 0 & 0 \\
\hline
\end{tabular}

Note: Vertical dashed lines represent median time to treatment discontinuation.

${ }^{a}$ Adjusted for age, sex, $\mathrm{CCl}$, region, and health care plan type.

$A L K=$ anaplastic lymphoma kinase; $C C I=$ Charlson Comorbidity Index; $H R=$ hazard ratio.

perform analyses; statistical significance was determined with a 2 -sided $\mathrm{P}<0.05$.

\section{Results}

In total, 1,540 unique patients were identified in the pooled dataset. The ALK inhibitor-naive cohort comprised 1,482 patients receiving alectinib $(n=445)$ or crizotinib $(n=1,037)$; the ALK inhibitor-pretreated cohort comprised patients receiving alectinib $(n=604)$, brigatinib $(n=142)$, or ceritinib $(n=134)$ as second or later ALK inhibitors (Table 1). In the ALK inhibitor-pretreated cohort, 755 patients were on a second ALK inhibitor, 139 were on a third ALK inhibitor, and 8 were on a fourth ALK inhibitor. Owing to small sample sizes, ALK inhibitor-naive patients who received ceritinib $(n=45)$ or brigatinib $(n=13)$ and ALK inhibitor-pretreated patients who received crizotinib $(n=21)$ were not included in the analyses.

In the ALK inhibitor-naive cohort, patients receiving alectinib were significantly younger than those receiving crizotinib (mean [SD] age: 59 [12] vs 61 [13] years; $P=0.002$ ), had a lower comorbidity burden (17\% vs $23 \%$ of patients with a CCI of at least 2, respectively; $\mathrm{P}=0.006$ ), and were more likely to be enrolled in employer-sponsored commercial health care insurance plans (32\% vs $28 \%$, respectively; $P=0.017$; Table 1). There were no significant differences in baseline characteristics between treatment groups in the ALK inhibitor-pretreated cohort (Table 1).

The analysis of adherence included 1,280 and 818 patients in the ALK inhibitor-naive (alectinib, $n=395$; crizotinib, $\mathrm{n}=885$ ) and ALK inhibitor-pretreated (alectinib, $\mathrm{n}=566$; brigatinib, $n=132$; ceritinib, $n=120$ ) cohorts, respectively, who received at least 2 prescriptions for ALK inhibitors. Adherence was high across all ALK inhibitors in both cohorts (Table 2).

Mean (SD) MPR for the ALK inhibitor-naive cohort over the entire treatment period was similar for alectinib and crizotinib (0.974 [0.084] vs 0.973 [0.098], respectively); the proportion of patients with an MPR of at least 0.8 was 95\% for both treatments in this cohort. Mean (SD) MPR and the proportion of patients with an MPR of at least 0.8 were similar for alectinib (0.958 [0.115]; 92\%), brigatinib (0.965 [0.122]; 93\%), and ceritinib (0.974 [0.089]; 93\%) in the ALK inhibitor-pretreated cohort. Adherence to alectinib 


\section{FIGURE 3 Time to Treatment Discontinuation With Alectinib, Brigatinib, or Ceritinib in the ALK Inhibitor-Pretreated Cohort}

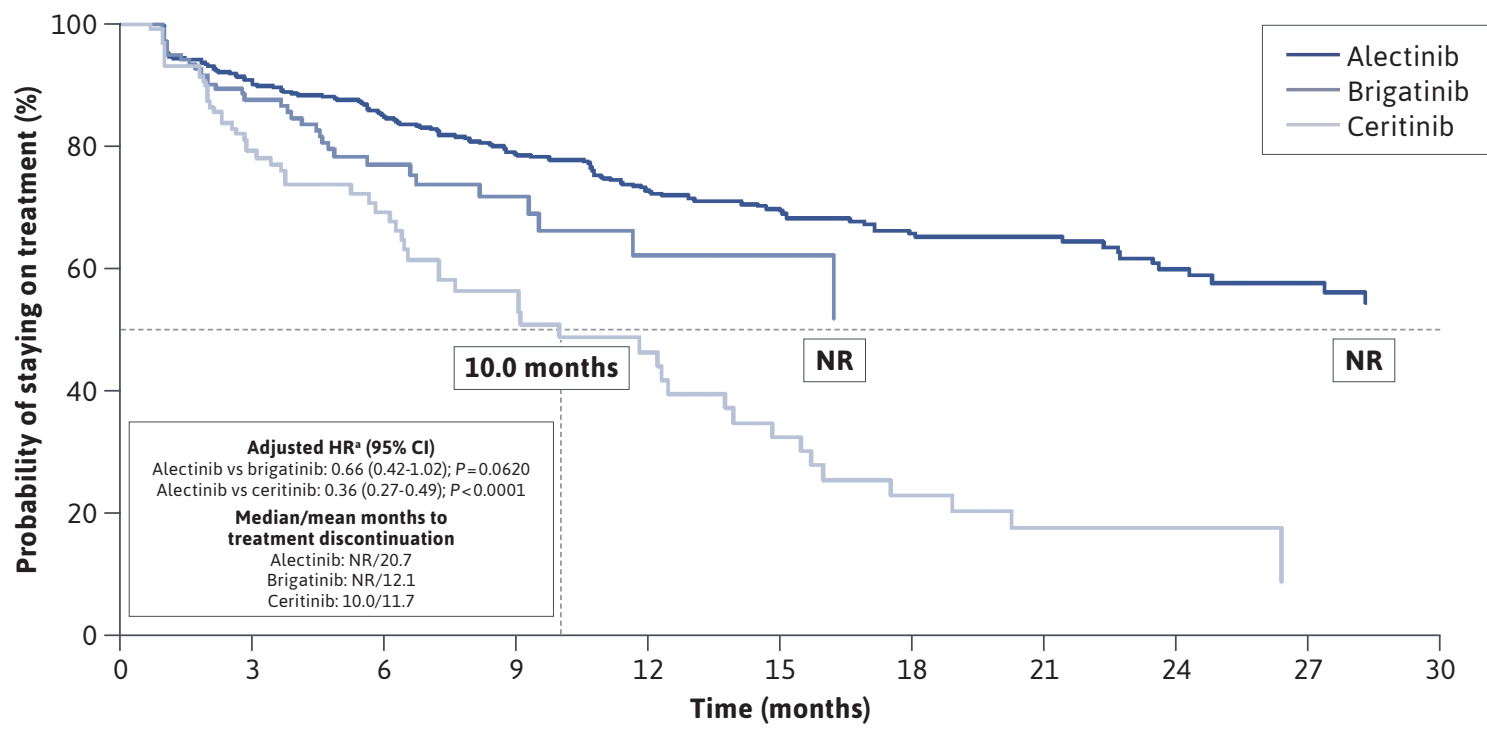

Patients on treatment ( $\mathrm{n})$

\begin{tabular}{l|c|c|c|c|c|c|c|c|c|c|c}
\hline Alectinib & 604 & 503 & 397 & 292 & 227 & 182 & 118 & 116 & 62 & 52 & 30 \\
\hline Brigatinib & 142 & 103 & 60 & 35 & 15 & 15 & 5 & 0 & 0 & 0 & 0 \\
\hline Ceritinib & 134 & 82 & 47 & 34 & 20 & 14 & 9 & 6 & 6 & 1 & 0 \\
\hline
\end{tabular}

Note: Vertical dashed lines represent median time to treatment discontinuation.

${ }^{a}$ Adjusted for age, sex, $\mathrm{CCl}$, region, health care plan type, and ALK inhibitor sequence.

$A L K=$ anaplastic lymphoma kinase; $C C I=C h a r l s o n$ Comorbidity Index; $H R=$ hazard ratio; $N R=$ not reached.

in the ALK inhibitor-naive cohort was significantly higher at 6 months than to crizotinib among patients with at least 6 months of post-index enrollment/activity (alectinib, $\mathrm{n}=330,79.1 \%$; crizotinib, $\mathrm{n}=658,67.0 \%$; IRR [95\% CI]: 1.19 [1.02-1.39]) after adjustment for patient characteristics.

Unadjusted median persistence (time to treatment discontinuation) was 27.1 months for alectinib vs 8.8 months for crizotinib in the ALK inhibitor-naive cohort. After adjusting for baseline characteristics, patients receiving alectinib in this cohort were $46 \%$ less likely to discontinue treatment than patients receiving crizotinib (aHR [95\% CI]: 0.54 [0.440.65 ]; $P<0.0001$; Figure 2). In the sensitivity analysis of time to next ALK inhibitor, patients receiving alectinib were $77 \%$ less likely to switch ALK inhibitors than patients receiving crizotinib (aHR [95\% CI]: 0.23 [0.17-0.33]; P<0.0001); median time to switching was 24.5 months for crizotinib vs not reached for alectinib (only 19.2\% of patients receiving alectinib switched to another ALK inhibitor at 24.5 months; Supplementary Figure 2, available in online article).
In the ALK inhibitor-pretreated cohort, patients receiving alectinib were $64 \%$ less likely to discontinue treatment than patients receiving ceritinib (aHR [95\% CI]: 0.36 [0.27-0.49]; $\mathrm{P}<0.0001$; Figure 3 ). In this cohort, the risk of discontinuation for alectinib was $34 \%$ lower than for brigatinib but was not statistically significant (aHR [95\% CI]: 0.66 [0.42-1.02]; $\mathrm{P}=0.062$; Figure 3 ). Although the treatment discontinuation analysis using a 30-day gap yielded similar findings to the analysis using a 60-day gap, patients who received alectinib were significantly less likely to discontinue treatment than those who received brigatinib (aHR [95\% CI]: 0.54 [0.360.79]; $P=0.0015$; Supplementary Figures 3 and 4 , available in online article).

\section{Discussion}

This study provided evidence of longer real-world persistence with alectinib than with other evaluated ALK inhibitors in ALK inhibitor-naive and ALK inhibitor- 
pretreated patients with NSCLC using pooled US insurance claims data. High adherence to treatment was observed in both ALK inhibitor-naive and ALK inhibitor-pretreated cohorts for all evaluated ALK inhibitors.

Our findings of high adherence during the treatment period for all ALK inhibitors suggest that differences in dosing schedules and pill burdens may have little impact on real-world treatment adherence for this patient population. However, other factors that are not measured in this study may also influence adherence. These include disease severity and adverse events, as well as patient-related factors such as quality of life, out-of-pocket expenses, beliefs about disease or medication, and behavioral or social characteristics. ${ }^{21-23}$

In oncology, many treatments are indicated for use until the time of disease progression, as long as the patient can tolerate the medicine; hence, low time to treatment discontinuation is usually an indication of either disease progression or of unacceptable side effects. Time to discontinuation could therefore provide insights into how trial-observed progression-free survival (PFS) translates to the real-world setting. ${ }^{17}$ Furthermore, persistence reflects both safety and effectiveness, as well as nonclinical factors such as cost and patient preference, thereby providing a more holistic view of treatment utilization in the real world than evaluation of PFS (which only measures efficacy) in a clinical trial setting. Longer persistence was observed in ALK inhibitor-naive patients treated with alectinib than in those treated with crizotinib. This finding complements results from the ALEX, J-ALEX, and ALESIA trials, which showed that alectinib was associated with longer PFS and lower toxicity than crizotinib in treatment-naive patients with ALK-positive NSCLC. 16,24,25

In the ALK inhibitor-pretreated cohort, alectinib had significantly longer persistence than ceritinib. In the same cohort, a 34\% lower likelihood of discontinuation was observed for alectinib than for brigatinib, with the difference in persistence approaching statistical significance $(P=0.062)$. It is possible that the lack of a statistically significant result may be due to small sample sizes and to the shorter follow-up with brigatinib than with alectinib (Table 2). However, the results of the sensitivity analysis, which used a more stringent treatment discontinuation definition (30-day gap), showed that persistence with alectinib was significantly higher than with brigatinib, further indicating that additional follow-up time to allow the occurrence of discontinuation may confirm the main results. For ALK inhibitor-pretreated patients, these realworld persistence findings allow us to make a holistic comparison across ALK inhibitors, whereas cross-trial indirect comparisons of multiple single-arm phase $1 / 2$ trials, such as NP28761 and NP28673 for alectinib and ALTA for brigatinib, would be difficult and inappropriate. ${ }^{26}$ Given the availability of multiple ALK inhibitors, further research is warranted to understand the factors that affect realworld adherence and persistence and the optimal sequence of use.

To our knowledge, this is the first study to estimate adherence and persistence with ALK inhibitors using realworld claims data. The pooling of 3 claims data sources provided a larger sample size, which enabled evaluation of treatments in a rare disease population. However, for newer ALK inhibitors such as brigatinib, a larger sample size and longer follow-up time would improve our ability to interpret the findings.

\section{LIMITATIONS}

This study is subject to the inherent limitations of retrospective analyses of secondary data, including missing data and coding errors. ${ }^{27}$ Specifically, administrative claims data only capture whether a prescription was filled and cannot determine whether a patient took the medication as prescribed. Clinical parameters such as disease severity and line of therapy, as well as biomarker test results, were not captured in claims, and other unobserved factors such as out-of-pocket costs and incomes were unavailable to permit comprehensive model adjustments. Specifically, crizotinib is also indicated for the treatment of ROS1-positive metastatic NSCLC. As ROS1-positive NSCLC is a rare condition, ${ }^{28-30}$ prescriptions for this indication would not be considered to represent a significant proportion of patients receiving crizotinib.

In addition, inclusion of ROS1-positive crizotinib patients in this study would result in an overestimation of crizotinib treatment duration because ROS1-positive NSCLC patients have better PFS compared with patients with ALK-positive NSCLC (median PFS: ROS1-positive=19.2 months; ALKpositive $=10.9$ months) when treated with crizotinib in clinical trials. ${ }^{31,32}$ The analysis of alectinib vs crizotinib in the ALK inhibitor-naive cohort was considered conservative, yet still demonstrates that alectinib had largely improved persistence.

A specific limitation of the IDV database is that it is a provider-based, open-claims system without patient enrollment records; therefore, claims for patients who switch pharmacies and/or receive care from providers not covered by IDV would not have been captured; however, it should be noted that this study utilized claims activities as a surrogate of continuous enrollment to help mitigate the limitation in the open-claims database. Although this is unlikely to be the case for patients with NSCLC who are receiving specialty care, patients in the IDV were required 
to have both medical and pharmacy claims activities in both pre-index and post-index periods to ensure that they stayed within provider networks.

ALK inhibitor cohorts were categorized by sequence of use (ALK inhibitor-naive and ALK inhibitorpretreated) rather than by line of therapy (first line, second line, and beyond), because the use of infusion chemotherapy was not captured in pharmacy claims. This could potentially result in an overestimation of the time to next ALK inhibitor. Such overestimation was mitigated by limiting the time to next ALK inhibitor analysis to the ALK inhibitor-naive cohort. In clinical practice, it is common for clinicians to prescribe a different, second ALK inhibitor if a patient's disease progresses during treatment with the first ALK inhibitor, whereas patients whose disease does not respond to multiple different ALK inhibitors are often switched to chemotherapy.

\section{Conclusions}

In the real-world setting, alectinib was associated with longer treatment persistence than crizotinib in ALK inhibitor-naive patients and ceritinib in ALK inhibitor-pretreated patients. Persistence with alectinib was observed to be directionally higher than for brigatinib in the ALK inhibitor-pretreated cohort, and this finding approached statistical significance. Adherence was high and similar across all ALK inhibitors in both the ALK inhibitor-naive and ALK inhibitor-pretreated cohorts. Further research with more patients over a longer period of time will be valuable in evaluating real-world clinical outcomes in the context of ALK inhibitor treatment sequences, adherence, and persistence.

\section{DISCLOSURES}

This study was funded by Genentech Inc. Ganti has received research support from Takeda and has provided consulting services to Genentech Inc., AstraZeneca, Flagship Biosciences, Cardinal Health, BioGene, Mirati Therapeutics, Blueprint Medicines, and G1 Therapeutics. Lin, Wong, and Ogale are employees of Genentech Inc. and may own stock in F. Hoffmann-La Roche. Yang was employed by Genentech Inc. at the time of this study.

Part of the study findings were presented as a poster at the NCCN 2020 Virtual Annual Conference, April 9, 2020.

\section{ACKNOWLEDGMENTS}

Medical writing support was provided by Harriet Bell of PharmaGenesis London, London, UK, and Rebecca Hornby, PhD, of Oxford PharmaGenesis, Oxford, UK, and funded by Genentech Inc.

\section{REFERENCES}

1. Torre LA, Siegel RL, Jemal A. Lung cancer statistics. Adv Exp Med Biol. 2016;893:1-19.

2. American Cancer Society. Cancer facts and figures 2019. Accessed May 5, 2020. https://www.cancer.org/content/dam/cancer-org/research/ cancer-facts-and-statistics/annualcancer-facts-and-figures/2019/ cancer-facts-and-figures-2019.pdf

3. Herbst RS, Heymach JV, Lippman SM. Lung cancer. N Engl J Med. 2008;359(13): 1367-80

4. Horn L, Pao W. EML4-ALK: honing in on a new target in non-small-cell lung cancer. J Clin Oncol. 2009;27(26):4232-35.

5. Johnson BE, Kris MG, Berry LD, et al. A multicenter effort to identify driver mutations and employ targeted therapy in patients with lung adenocarcinomas: the Lung Cancer Mutation Consortium (LCMC). J Clin Oncol. 2013;31(15_suppl):8019.

6. Simeone JC, Nordstrom BL, Patel K, et al. Treatment patterns and overall survival in metastatic non-small-cell lung cancer in a real-world, US setting. Future Oncol. 2019;15:3491-502.
7. National Cancer Institute. SEER cancer statistics factsheets: lung and bronchus cancer. 2015. Accessed January 13, 2020. http://seer.cancer.gov/statfacts/html/ lungb.html

8. Kazandjian D, Blumenthal GM, Chen HY, et al. FDA approval summary: crizotinib for the treatment of metastatic non-small cell lung cancer with anaplastic lymphoma kinase rearrangements. Oncologist. 2014;19(10):e5-11.

9. Carlson JJ, Suh K, Orfanos P, et al. Cost effectiveness of alectinib vs. crizotinib in first-line anaplastic lymphoma kinase-positive advanced non-smallcell lung cancer. Pharmacoeconomics. 2018;36(4):495-504.

10. Doebele RC, Pilling AB, Aisner DL, et al. Mechanisms of resistance to crizotinib in patients with ALK gene rearranged nonsmall cell lung cancer. Clin Cancer Res. 2012;18(5):1472-82.

11. Costa DB, Shaw AT, Ou SH, et al. Clinical experience with crizotinib in patients with advanced ALK-rearranged non-small-cell lung cancer and brain metastases. J Clin Oncol. 2015;33(17): 1881-88.

12. Beardslee T, Lawson J. Alectinib and brigatinib: new second-generation ALK inhibitors for the treatment of nonsmall cell lung cancer. J Adv Pract Oncol. 2018;9(1):94-101.

13. Pirker R, Filipits M. From crizotinib to lorlatinib: continuous improvement in precision treatment of ALK-positive non-small cell lung cancer. ESMO Open. 2019;4:e00548.

14. Mok T, Camidge DR, Gadgeel SM, et al. Updated overall survival and final progression-free survival data for patients with treatment-naive advanced ALKpositive non-small-cell lung cancer in the ALEX study. Ann Oncol. 2020;31:1056-64.

15. Nakagawa K, Hida T, Nokihara H, et al. Final progression-free survival results from the J-ALEX study of alectinib versus crizotinib in ALK-positive non-small-cell lung cancer. Lung Cancer. 2020;139:195-99. 
16. Zhou C, Kim SW, Reungwetwattana T, et al. Alectinib versus crizotinib in untreated Asian patients with anaplastic lymphoma kinase-positive non-smallcell lung cancer (ALESIA): a randomised phase 3 study. Lancet Respir Med. 2019;7(5):437-46.

17. Blumenthal GM, Gong Y, Kehl K, et al. Analysis of time-to-treatment discontinuation of targeted therapy, immunotherapy, and chemotherapy in clinical trials of patients with non-small-cell lung cancer. Ann Oncol. 2019;30:830-38.

18. Goren A, DiBonaventura M, Wagner R. Adherence and its association with health outcomes among patients currently treated for leukemia, melanoma, or nonsmall cell lung cancer (NSCLC). Value Health. 2013;16:A144-A145.

19. Cramer JA, Roy A, Burrell A, et al. Medication compliance and persistence: terminology and definitions. Value Health. 2008;11(1):44-47.

20. Zou G. A modified poisson regression approach to prospective studies with binary data. Am J Epidemiol. 2004;159(7): 702-06.

21. Timmers L, Boons CC, Mangnus D, et al. The use of erlotinib in daily practice: a study on adherence and patients' experiences. BMC Cancer. 2011;11:284.
22. Hess LM, Louder A, Winfree K, et al. Factors associated with adherence to and treatment duration of erlotinib among patients with non-small cell lung cancer. J Manag Care Spec Pharm. 2017;23:643-52. doi:10.18553/jmcp.2017.16389

23. Seetasith A, Wong W, Tse J, et al. The impact of copay assistance on patient out-of-pocket costs and treatment rates with ALK inhibitors. J Med Econ. 2019;22(5):414-20.

24. Peters S, Camidge DR, Shaw AT, et al. Alectinib versus crizotinib in untreated ALK-positive non-small-cell lung cancer. N Engl J Med. 2017;377(9):829-38.

25. Hida T, Nokihara H, Kondo M, et al. Alectinib versus crizotinib in patients with ALK-positive non-small-cell lung cancer (J-ALEX): an open-label, randomised phase 3 trial. Lancet. 2017;390(10089):29-39.

26. Reckamp K, Lin HM, Huang J, et al. Comparative efficacy of brigatinib versus ceritinib and alectinib in patients with crizotinib-refractory anaplastic lymphoma kinase-positive non-small cell lung cancer. Curr Med Res Opin. 2019;35(4):569-76.
27. Fang J, Bruce Wirta S, Kahler K. Secondary use of data: non-interventional study best practices in planning and protocol development. J Health Econ Outcomes Res. 2017;5(1):27-38.

28. Zugazagoitia J, Molina-Pinelo S, Lopez-Rios F, et al. Biological therapies in non-small cell lung cancer. Eur Respir J. 2017;49(3):1601520.

29. Clave S, Gimeno J, Munoz-Marmol AM, et al. ROS1 copy number alterations are frequent in non-small cell lung cancer. Oncotarget. 2016;7(7):8019-28.

30. Scheffler M, Schultheis A, Teixido C, et al. ROS1 rearrangements in lung adenocarcinoma: prognostic impact, therapeutic options and genetic variability. Oncotarget. 2015;6(12):10577-85.

31. Shaw AT, Riely GJ, Bang YJ, et al. Crizotinib in ROS1-rearranged advanced non-small-cell lung cancer (NSCLC): updated results, including overall survival, from PROFILE 1001. Ann Oncol. 2019;30(7):1121-26.

32. Solomon BJ, Kim DW, Wu YL, et al. Final overall survival analysis from a study comparing first-line crizotinib versus chemotherapy in ALK-mutation-positive non-small-cell lung cancer. J Clin Oncol. 2018;36(22):2251-58. 